\title{
Overexpression of Tyro3 and its implications on hepatocellular carcinoma progression
}

\author{
YAN DUAN $^{1 *}$, WINNIE WONG $^{1 *}$, SONJA COURTNEY CHUA $^{1}$, HWEE LIN WEE ${ }^{1}$, \\ SENG GEE LIM ${ }^{3}$, BOON TIN CHUA ${ }^{2}$ and HAN KIAT HO ${ }^{1}$ \\ ${ }^{1}$ Department of Pharmacy, Faculty of Science, National University of Singapore, Singapore 117543; \\ ${ }^{2}$ Institute of Molecular and Cell Biology, Agency for Science Technology and Research, Singapore 138673; \\ ${ }^{3}$ Department of Gastroenterology and Hepatology, National University Health System, \\ Singapore 119228, Republic of Singapore
}

Received September 9, 2015; Accepted October 20, 2015

DOI: 10.3892/ijo.2015.3244

\begin{abstract}
While various tyrosine kinases have been associated with the pathogenesis of hepatocellular carcinoma (HCC), the identification of a dominant therapeutic target among them remains a challenge. Here, we investigated the role of Tyro3, a relatively uncharacterized member of the TAM (Tyro3, Axl and Mer) receptor family. The present study aimed to profile and identify potential association between Tyro3 expression in HCC and cancer phenotypes. RNAs obtained from 55 HCC patients were quantified for Tyro 3 expression in both cancerous tissue and the adjacent normal tissue. Expression profile was correlated with clinical data. These observations were further substantiated with in vitro $\mathrm{HCC}$ cell culture investigations. Tyro3 was strongly upregulated ( $>2$-fold elevation) in the tumor tissue of $\sim 42 \%$ of the patients. It was shown that higher expression level of Tyro3 was associated with the key tumor marker AFP, and the tumor diameter and liver injury marker ALT. Subsequent cell culture models indicated high expression in various HCC cell lines, in particular Hep3B. Gene silencing of Tyro3 in Hep3B effectively reduced cell proliferation, ERK phosphorylation and cyclin D1 expression, indicating a key in maintaining the proliferative state of these cells. Notably, silencing also suppressed the transcriptional and translational
\end{abstract}

Correspondence to: Dr Han Kiat Ho, Department of Pharmacy, Faculty of Science, National University of Singapore, 18 Science Drive 4, 117543 Singapore

E-mail:phahohk@nus.edu.sg

${ }^{*}$ Contributed equally

Abbreviations: AFP, $\alpha$-fetoprotein; ALT, alanine transaminase; AST, aspartate aminotransferase; GAPDH, glyceraldehyde 3phosphate dehydrogenase; HBV, hepatitis B virus infection; HCC, hepatocellular carcinoma; siRNA, small interfering ribonucleic acid; RTK, receptor tyrosine kinase; TAM, Tyro3, Axl and Mer

Key words: Tyro3, tyrosine kinase, hepatocellular carcinoma, $\alpha$-fetoprotein, cell proliferation expression of HCC tumor marker AFP. Overall, these data suggest that Tyro3 contributes significantly to tumor growth, aggressiveness and liver dysfunction. Inhibition of Tyro3 and its aberrant signaling in tumors with high expression could present new opportunities for HCC treatment.

\section{Introduction}

The lack of effective treatment against hepatocellular carcinoma (HCC), the fifth most common malignancy and the third leading cause of cancer deaths worldwide calls for directed efforts to better understand the disease and to identify new drug targets (1). Amidst the advent of molecular targeted therapy in cancer treatment, multi-kinase inhibitor sorafenib emerged as the first of such agents for use against HCC (2). This breakthrough creates the headway for more intense efforts at exploring different tyrosine kinases to mitigate or reverse the course of disease progression.

While several investigational tyrosine kinase inhibitors are in various stages of clinical development as therapeutics for HCC, only a small subset of the more than 90 tyrosine kinases has been targeted. Some notable kinases include EGFR, VEGFR, PDGFR, IGF1R and MET (3-5). Even so, response rates of $\mathrm{HCC}$ in clinical studies involving such targets remain equivocal. Apart from sorafenib, which has demonstrated the most significant improvement in overall survival (2), other FDA-approved tyrosine kinase inhibitors such as imatinib and vandetanib showed little therapeutic benefit $(6,7)$. The efficacy of sunitinib was also compromised by dose-limiting toxicities (8). These suboptimal outcomes could arise from an incomplete understanding of the roles of tyrosine kinases in HCC progression. Hence, we reasoned that broadening the scope of investigation by considering other proto-oncogenic tyrosine kinases will help uncover other important targets and establish any mechanism of HCC-specific relevance. The TAM (Tyro3, Axl and Mer) receptors became the focus of the present study, given recent reports associating them with various malignancies (9). TAM is a subfamily of receptor tyrosine kinases that signals through the binding to Gas6/Protein S. Axl and Mer overexpression has been reported in various tumors and some Axl inhibitors 
are already in development (10). Tyro3 is the least characterized member in the family. Its oncogenic potential was first demonstrated in murine models where high expression was observed in mammary tumors and transient transfection can result in development and growth of tumors (11). Other studies also suggested its role in multiple myeloma and lung carcinoma cell lines $(12,13)$. However, mechanistic insights and strong clinical data in support of its oncogenic role are lacking. Importantly, a recent in-house kinase inhibitor screen whereby an inhibitor of Tyro3 demonstrated selective and potent inhibition of HCC cell growth drew fresh evidence to justify Tyro3 as a novel target (14). Therefore, we embarked on this study to determine the clinical relevance of Tyro3 to $\mathrm{HCC}$, by associating its expression to clinical data; as well as to provide mechanistic evidence for any observed effects, through in vitro $\mathrm{HCC}$ cell culture model system.

In the present study, we demonstrate that Tyro3 is significantly overexpressed in $\sim 40 \%$ of HCC patients. Furthermore, expression level was associated with the severity of liver injury and disease progression. Cell-based models were able to draw important evidence that Tyro3 plays a major role in regulating cell proliferation and AFP production, encapsulating a potentially important biological response that will support the development of a HCC-targeted therapeutic strategy.

\section{Materials and methods}

Cell culture. Hep3B, HepG2, SK-Hep1 and PLC-PRF/5 and were purchased from the American Type Culture Collection (ATCC; Manassas, VA, USA) while Hs817T, Hs1.Li and Huh7 were part of an in-house collection through Professor Axel Ullrich (Department of Molecular Biology, Max Planck Institute of Biochemistry, Martinsried, Germany). Hep3B, HepG2 and PLC-PRF/5 were maintained in minimal essential medium (MEM) supplemented with $10 \%$ fetal bovine serum (FBS), sodium pyruvate $(1 \mathrm{mM})$ and non-essential amino acids (1X), and the rest in Dulbecco's modified Eagle's medium (DMEM) with $10 \%$ FBS and sodium pyruvate. Cells were maintained at $37^{\circ} \mathrm{C}$ in $5 \% \mathrm{CO}_{2}$. Unless stated otherwise, all cell culture reagents were obtained from Invitrogen (Life Technologies, Grand Island, NY, USA) and the chemicals from Sigma Aldrich (St. Louis, MO, USA).

HCC patient samples. These samples were obtained with appropriate approval from the Institutional Review Board of the National University Hospital System (NUHS). Total RNA was isolated from paired normal and HCC liver tissues from HCC patients $(n=55)$ at the NUHS as previously described (15). cDNAs were synthesized from $2 \mu \mathrm{g}$ RNA using SuperScript III reverse transcriptase kit (Invitrogen, Singapore, Singapore), performed according to the manufacturer's instructions and as briefly described in a later section.

HCC tissue microarray. The tissue microarray (TMA) slides were purchased from Cybrdi, Inc. (Rockville, MD, USA). Dako EnVision System-HRP (DAB) K 4010 was used for immunohistochemical staining. The slides were deparaffinized by washing 3 times in xylene. They were further rehydrated in $100 \%$ ethanol (3 times) and sequentially in 90, 80 and $70 \%$ ethanol v/v (each $3 \mathrm{~min}$ ), followed by washing under running water for $1 \mathrm{~min}$. All incubations were performed at room temperature unless otherwise stated. The slides were then blocked in $1 \%$ hydrogen peroxide in methanol for $30 \mathrm{~min}$ to block endogenous peroxidase. Following washing with water, antigen retrieval was performed with Agilent Dako target retrieval solution at pH 6.0 (Agilent Technologies Carpinteria, CA, USA) in a pressure cooker for $20 \mathrm{~min}$. After cooling and washing, the slides were blocked with $10 \%$ goat serum in PBS for $20 \mathrm{~min}$. This was followed by incubation in the Tyro3 antibody (1:50) in 10\% goat serum and PBS for $2 \mathrm{~h}$. After washing with PBS, secondary antibody solution of anti-rabbit horseradish peroxidase (HRP)-conjugated antibody (Dako) was added to each slide and left to incubate for $30 \mathrm{~min}$ in the dark. Slides were washed and followed by addition of $200 \mu \mathrm{l}$ chromogen-substrate solution ( $1 \mathrm{ml}$ buffer +1 drop of DAB) to each slide. The reaction was stopped by adding water. Nuclear staining included hematoxylin for $5 \mathrm{~min}$, washing under running water for $30 \mathrm{sec}$, dipping in acid alcohol for $15 \mathrm{sec}$, washing and in Scott's tap water for $15 \mathrm{sec}$. The slides were then dehydrated for $3 \mathrm{~min}$ in increasing percentages of ethanol (70, 80 and 90\%), cleared in xylene and coverslips were mounted with Cytoseal from Electron Microscopy Sciences (Hatfield, PA, USA). The results were analyzed under microscopy. All chemicals were obtained from Sigma-Aldrich unless otherwise stated. The rabbit polyclonal Tyro3 IHC antibody used to detect Tyro3 expression was obtained from Bethyl Laboratories (Montgomery, TX, USA).

cDNA synthesis by reverse transcription. For single strand cDNA synthesis, specified amount of total RNA, primer, dNTP mix $(10 \mathrm{mM})$, diethylpyrocarbonate (DEPC)-treated water in a total volume of $10 \mathrm{ml}$ was prepared. The reaction mix was incubated at $65^{\circ} \mathrm{C}$ for $5 \mathrm{~min}$, and at least at $4^{\circ} \mathrm{C}$ for $5 \mathrm{~min}$ for the annealing of the Oligo-dT primers to the mRNAs. After that, the tubes were taken out and put on ice, and $10 \mu \mathrm{l}$ of synthesis mix was added in the system. The synthesis mix contained $2.0 \mu \mathrm{l}$ of $10 \mathrm{X}$ buffer, $4 \mu \mathrm{l}$ of $\mathrm{MgCl}_{2}(25 \mathrm{mM}), 2 \mu \mathrm{l}$ dithiothreitol (DTT) $(0.1 \mathrm{M}), 1 \mu \mathrm{l}$ RNase OUT (40 U/ $\mu \mathrm{l}), 1 \mu \mathrm{l}$ SuperScript III RT $(200 \mathrm{U} / \mu \mathrm{l})$. Then $10 \mu \mathrm{l}$ of synthesis mix was added into the prior reaction mix, which was then incubated for $50 \mathrm{~min}$ at $50^{\circ} \mathrm{C}$ followed by a termination of reaction at $85^{\circ} \mathrm{C}$ for $5 \mathrm{~min}$ and cooled to $4^{\circ} \mathrm{C}$. The, $1 \mu \mathrm{l}$ of RNase $\mathrm{H}$ was added and incubated at $37^{\circ} \mathrm{C}$ for $20 \mathrm{~min}$. The tubes were stored at $-20^{\circ} \mathrm{C}$ until further usage.

Quantitative RT-PCR. Quantitative RT-PCR was carried out using an Applied Biosystems 7300 Real-time PCR system (Applied Biosystems, Foster City, CA, USA) with pre-optimized TaqMan gene expression assay for human Tyro3 (NM_006293) and $\beta$-actin as the housekeeping control. The thermal cycling condition comprised an initial denaturation step at $95^{\circ} \mathrm{C}$ for $10 \mathrm{~min}$, followed by 40 cycles at $95^{\circ} \mathrm{C}$ for $15 \mathrm{sec}$ and $60^{\circ} \mathrm{C}$ for $60 \mathrm{sec}$. The samples were prepared in triplicate with $4 \mathrm{ml}$ of prediluted cDNA (2-10-fold) samples each. For additional Tyro3 transcript quantification in HCC cell lines as well as for gene silencing experiments, SYBRbased assay was performed using primers purchased from 1st BASE Pte Ltd. (Singapore, Singapore). Here, forward primer for Tyro3 was 5'-CGGTAGAAGGTGTGCCATTT-3' and reverse primer 5'-TGGGTCACCCCTGTTACATT-3'. Forward 


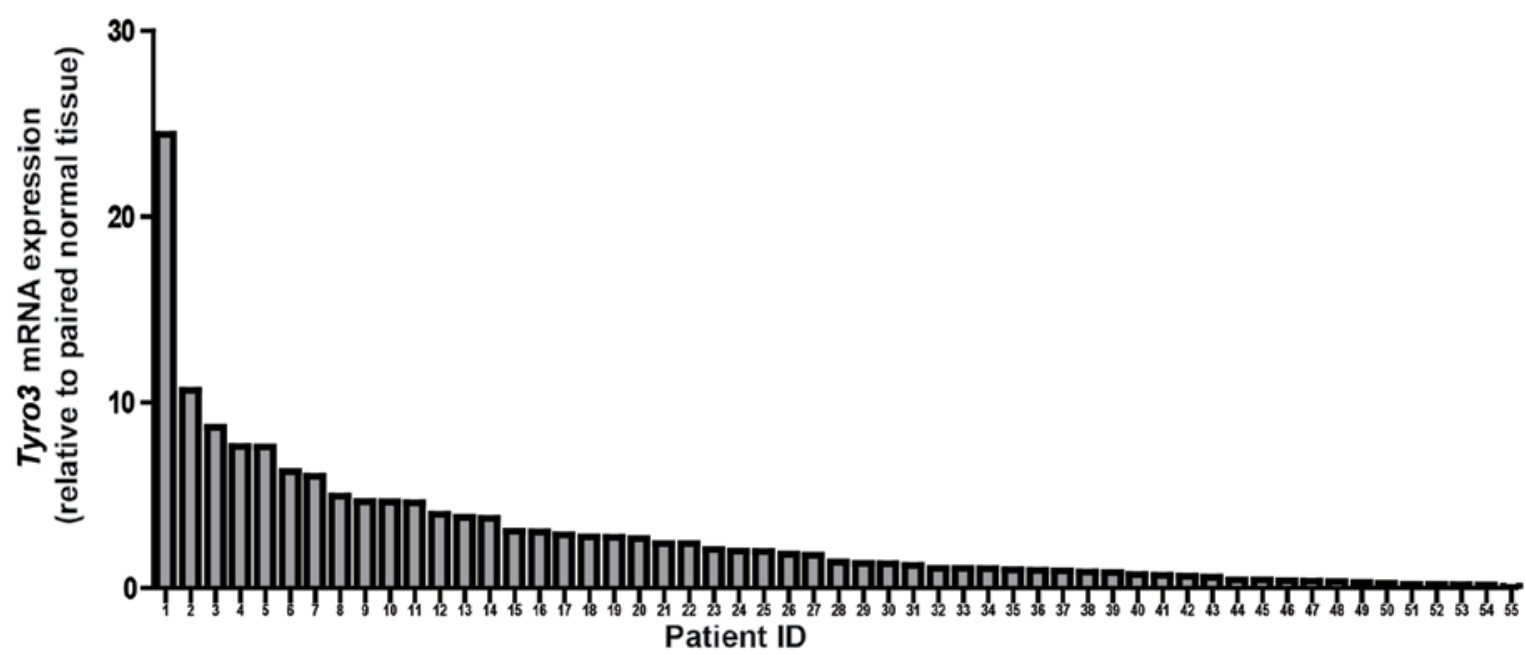

Figure 1. Tyro3 expression is upregulated in human liver tumors. Analysis of Tyro3 gene expression using qRT-PCR in tumor tissue expressed as fold change relative to its matched normal tissue after normalizing to $\beta$-actin in the $55 \mathrm{HCC}$ patients. The expression profile is ordered and presented from the highest to the lowest Tyro3 expressor (left to right).

primer for Afp was 5'-AGCTTGGTGGTGGATGAAAC-3' and reverse primer 5'-TCTTGCTTCATCGTTTGCAG-3'. $G A P D H$ was used as housekeeping control with forward primer 5'-ATGTTCGTCATGGGTGTGAA-3' and reverse primer 5'-TGTGGTCATGAGTCCTTCCA-3'. For PCR reaction, each well contained $4 \mu \mathrm{l}$ synthesized cDNA, $2 \mu \mathrm{l}$ each of $10 \mu \mathrm{M}$ forward and reverse primers, $2 \mu \mathrm{l}$ nuclease-free water and $10 \mu 1$ Power SYBR-Green PCR Master Mix (Applied Biosystems). Data were obtained as an average $C_{T}$ value, and subsequently normalized against respective endogenous control as $\Delta \mathrm{C}_{\mathrm{T}}$. Expression changes in Tyro3 transcripts between the normal and the corresponding tumor tissue were expressed as fold change using $2^{\text {(difference in } \Delta \mathrm{CT} \text { between pairs) }}$. For other in vitro experiments, expression was calculated similarly for difference in $\Delta \mathrm{C}_{\mathrm{T}}$ between $\mathrm{HCC}$ cell lines and noncancerous liver cell line.

Western blotting. All fractions collected were assayed for protein concentration using a BCA protein assay kit (Pierce, Rockford, IL, USA). Sample proteins (30-50 $\mu \mathrm{g}$ ) were resolved by denaturing electrophoresis using 7.5\% SDS-PAGE and transferred to nitrocellulose membranes for $2 \mathrm{~h}$ at $5 \mathrm{~V}$ using Trans-Blot SD Semi-Dry Transfer Cell (Bio-Rad Laboratories, Hercules, CA, USA). Immunodetection was by chemiluminescence (SuperSignal West Dura Extended; Pierce) using specific antibodies diluted in PBS with $0.05 \%$ (v/v) Tween-20 and 5\% (w/v) powdered milk. Primary antibodies used were anti-Tyro3 (H-110) from Santa Cruz Biotechnology (Santa Cruz, CA, USA), phospho-p44/42 ERK1/2, anti-phospho-Akt antibodies from Cell Signaling Technology (Beverly, MA, USA), HSP60 from Sigma Aldrich, anti- $\beta$-actin from Abcam (Cambridge, MA, USA) and cyclin D1 from Upstate (Lake Placid, NY, USA). Secondary anti-mouse and anti-rabbit horseradish peroxidase conjugated secondary antibodies (Pierce) were used at a dilution of 1:10,000.

Gene silencing by siRNA. Hep3B cells were grown to $50 \%$ confluence before transfection with siRNA (small interfering
RNAs) in 24-well plates. Custom-made ON-TARGETplus siRNA designed for silencing Tyro3 (Accession number: NM_002011) expression was purchased from Dharmacon (Chicago, IL, USA). A microcentrifuge tube containing $1.3 \mu \mathrm{l}$ of $20 \mu \mathrm{M}$ siRNA and $40.2 \mu \mathrm{l}$ growth medium was prepared (Tube A). Simultaneously, another tube containing $1 \mu 1$ Oligofectamine (Invitrogen) and $7.5 \mu \mathrm{l}$ growth medium was also prepared (Tube B). Both tubes were incubated at room temperature for $5 \mathrm{~min}$ before combining the contents and left to stand for another $20 \mathrm{~min}$. Next, each well of Hep3B cells was replaced with fresh serum-free medium $(200 \mu 1)$. The combined volume of the siRNA transfection mix $(50 \mu \mathrm{l})$ was added to the well and incubated at $37^{\circ} \mathrm{C}$. After $5 \mathrm{~h}$, the samples were loaded with another $250 \mu \mathrm{l}$ of growth medium containing $20 \%$ serum. Cells were cultured until the day of the assay.

Cell viability assay. Forty-eight hours post-transfection with Tyro3-targeting siRNA, eight replicates (7,500 cells/well) were seeded in a 96-well plate. After incubating for $24 \mathrm{~h}$, CellTiter-Glo luminescent assay (Promega, Madison, WI, USA) was used to measure ATP content as a gauge of the viable cell count, performed according to the manufacturer's instruction. ATP levels were determined and measured using SpectraMax M5 (Molecular Devices, Sunnyvale, CA, USA) and expressed in relative luminescence units (RLU).

Statistical analysis. To evaluate the association between patient demographic and clinical characteristics with levels of Tyro3 expression (low-to-moderate/ high), the Chi-square test was employed for categorical variables while the Student's t-test was employed for continuous variables in univariate analyses. In multivariate analyses to adjust for potential confounders, variables that are statistically significant at $\mathrm{P}<0.10$ in univariate analyses were entered into the model. The choice of $\mathrm{P}<0.10$ over $\mathrm{P}<0.05$ was to avoid missing potential association. Patient demographic variables studied are gender and age (as a continuous variable). All statistical analyses were performed in Stata version 10 for Mac (StataCorp., 
Table I. Tyro3 mRNA expression.

A, Tyro3 mRNA expression in HCC patients $(n=55)$

Tyro3 mRNA expression (tumor vs. normal)

\begin{tabular}{lcc}
\hline Downregulation & No significant difference & Upregulation \\
\hline$<0.5$ & $0.5-2.0$ & $>2.0$ \\
12 & 20 & 23 \\
$(21.8 \%)$ & $(36.4 \%)$ & $(41.8 \%)$ \\
\hline
\end{tabular}

B, Association of Tyro3 expression with demographic and clinical variables in univariate analyses

\begin{tabular}{|c|c|c|c|}
\hline \multirow{2}{*}{$\begin{array}{l}\text { Patient } \\
\text { characteristics }\end{array}$} & \multicolumn{2}{|c|}{$\begin{array}{l}\text { Tyro3 expression, } \mathrm{N}(\%) \\
\text { unless specified otherwise }\end{array}$} & \multirow[b]{2}{*}{ P-value } \\
\hline & Low-to-moderate & High & \\
\hline Gender & & & 0.587 \\
\hline Female & $7(22.6)$ & $4(16.7)$ & \\
\hline Male & $24(77.4)$ & $20(83.3)$ & \\
\hline Mean (SD) age & $60.4(13.82)$ & $55.5(13.20)$ & 0.193 \\
\hline HBV status & & & 0.345 \\
\hline Yes & $21(67.7)$ & $19(79.2)$ & \\
\hline No & $10(32.3)$ & $5(20.8)$ & \\
\hline AFP level & & & 0.001 \\
\hline Low & $23(74.2)$ & $7(30.4)$ & \\
\hline High & $8(25.8)$ & $16(69.6)$ & \\
\hline AST level & & & 0.058 \\
\hline Low & $17(58.6)$ & $7(31.8)$ & \\
\hline High & $12(41.4)$ & $15(68.2)$ & \\
\hline ALT level & & & 0.036 \\
\hline Low & $15(51.7)$ & $5(22.7)$ & \\
\hline High & $14(48.3)$ & $17(77.3)$ & \\
\hline AST/ALT ratio & & & 0.667 \\
\hline Low & $23(74.2)$ & $19(79.2)$ & \\
\hline High & $8(25.8)$ & $5(20.8)$ & \\
\hline Tumor size & & & 0.039 \\
\hline Small & $5(16.1)$ & 0 & \\
\hline Large & $26(83.4)$ & $24(100)$ & \\
\hline Tumor multiplicity & & & 0.824 \\
\hline Single & $19(61.3)$ & $14(58.3)$ & \\
\hline Multiple & $12(38.7)$ & $10(41.7)$ & \\
\hline Tumor histology & & & 0.146 \\
\hline Poor-moderate & $25(83.3)$ & $23(95.8)$ & \\
\hline Well & $5(16.7)$ & $1(4.2)$ & \\
\hline
\end{tabular}

Tyro3 mRNA expression in the tumor tissue of HCC patient samples were normalized to their respective adjacent normal tissue expression. Results were expressed as fold-change and sub-divided into 3 categories: downregulation, no significant change or upregulation as shown in the Table. ${ }^{a}$ Chi-square test for categorical variables and Student's t-test for continuous variables. HBV, hepatitis B virus infection; AFP, $\alpha$ fetoprotein; ALT, alanine aminotransferase; AST, aspartate aminotransferase. Cut-offs: high AFP, $\geq 500 \mathrm{ng} / \mathrm{ml}$; high AST, $\geq 40$ units/l; high ALT, $\geq 35$ units/l; high AST/ALT ratio: $\geq 2$ and large tumor size, $\geq 3 \mathrm{~cm}$.
College Station, TX, USA). Patient clinical variables studied are HBV infection status (yes/no), AFP levels (indicator of cancer aggressiveness; coded low/high: cut-off at $500 \mathrm{ng} / \mathrm{ml}$, which represents the upper limit of normal range), AST levels (indicator of liver injury; coded low/high: cut-off at 40 units/1, which represents the upper limit of normal range), ALT levels (indicator of liver injury coded low/high: cut-off at 35 units/l, which represents the upper limit of normal range), AST/ALT ratio (indicative of type of liver abnormality; coded low/high: cut-off at 2, with levels above this being suggestive of alcoholic hepatitis), tumor size (small/ large: largest diameter at a cut-off of $3 \mathrm{~cm}$ ), tumor multiplicity (single/multiple), tumor histology (poor-moderate/well: poorly-to-moderately differentiated or well differentiated), cirrhosis (yes/no) and vascular invasion (yes/no).

\section{Results}

Tyro3 expression in HCC patients. HCC tumor tissues and matched adjacent normal tissue was obtained from 55 HCC patients during resection. These samples were previously processed for RNA isolation and analyzed as part of an earlier study (15). In the present study, Tyro3 transcript expression was determined by TaqMan real-time PCR method and compared internally (i.e. matched tumor vs. tissue). Using a 2-fold elevation/suppression in tumor as a threshold for significance, we observed almost $42 \%$ of patients (23/55) exhibited overexpression of Tyro3 in the tumor tissues. This represents the largest subgroup of the HCC patients when categorized into $>2$-fold, $<0.5$-fold or 0.5 - to 2 -fold (Table IA). The highest fold change detected was $\sim 24$-fold in 1 patient. Seven out of 55 patients demonstrated overexpression of $>10$-fold (Fig. 1).

Clinical correlations of Tyro3 expression. A summary of patient information has been previously published (15). In univariate analyses, four parameters demonstrated a statistically significant correlation with Tyro3 $(\mathrm{P}<0.10)$. High Tyro3 expression was associated with higher AFP ALT, as well an increased tumor size at its largest dimension (Table IB). However, in multivariate analyses, only the association with AFP remained statistically significant [odds ratio (95\% confidence interval): 1.98 (0.56-3.40), $\mathrm{P}=0.006]$.

HCC tissue microarray. A commercially available HCC tissue microarray was used to investigate Tyro3 protein expression. There were varied levels of expression in the normal and cancerous tissues. Within the same tumor grade, there was a difference in Tyro3 expression which reflected no apparent correlation between tumor grades and staining intensity (Table II and Fig. 2C and D). However, a notable observation was the intense staining in the blood vessels of grade II tumors as compared to other liver tissue (Fig. 2). Upon closer examination, it was found that staining of blood cells contributed significantly to the stain pattern seen in the HCC tissue. The specificity of the antibody was confirmed as we examined the liver tissue and found that adjacent necrotic tissue (Fig. 2B and C) were not stained.

Tyro3 expression across liver cancer cell lines. While clinical correlations with Tyro3 expression are evident in HCC patients, 

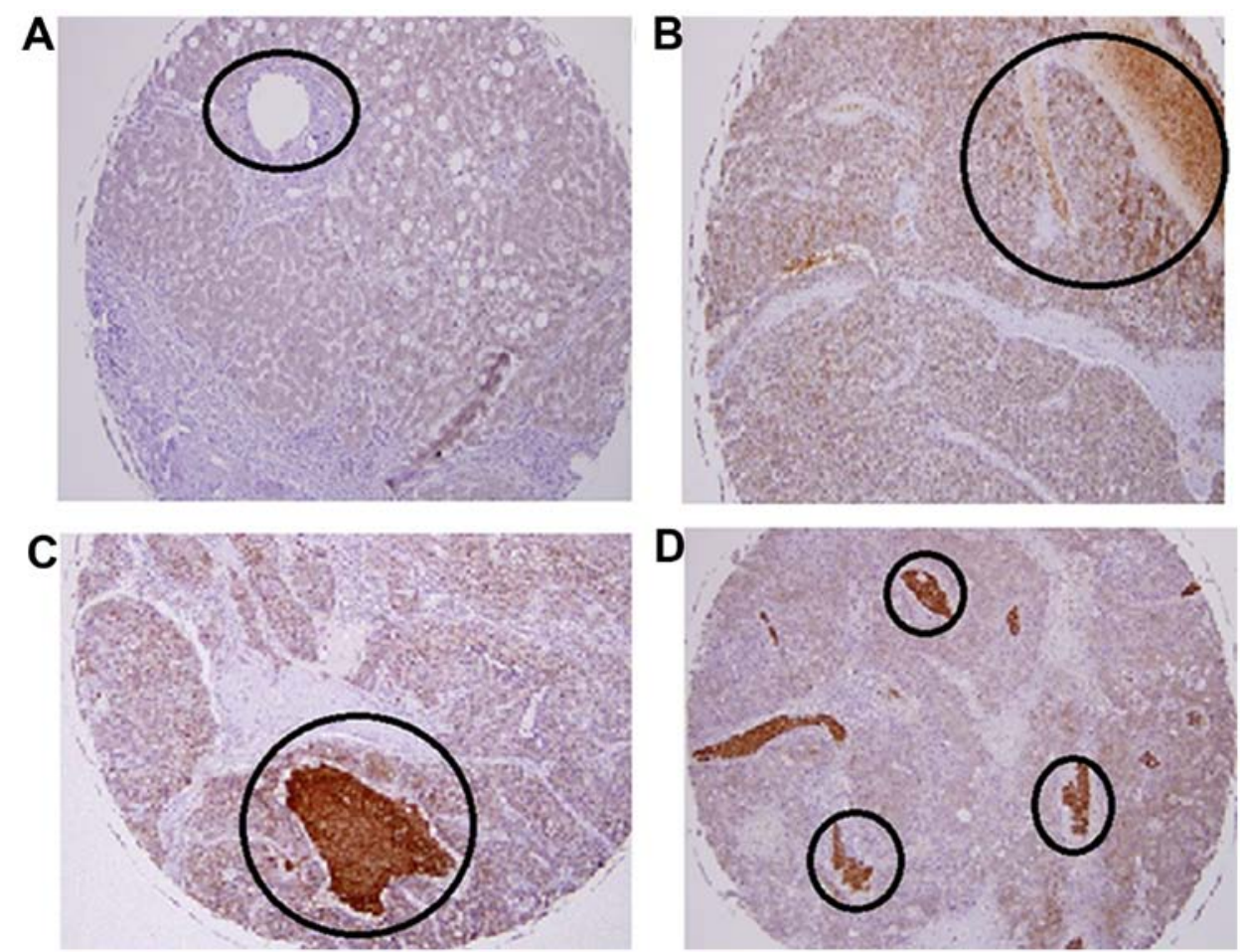

Figure 2. Immunohistological analysis of Tyro3 expression in HCC and control liver tissues. Immunostaining of liver tissue using Tyro3 antibody (Bethyl). Nuclear staining was revealed with haematoxylin. (A) Vacuolation. (B-D) HCC grade II. Circled are blood vessels (Magnification, x100).
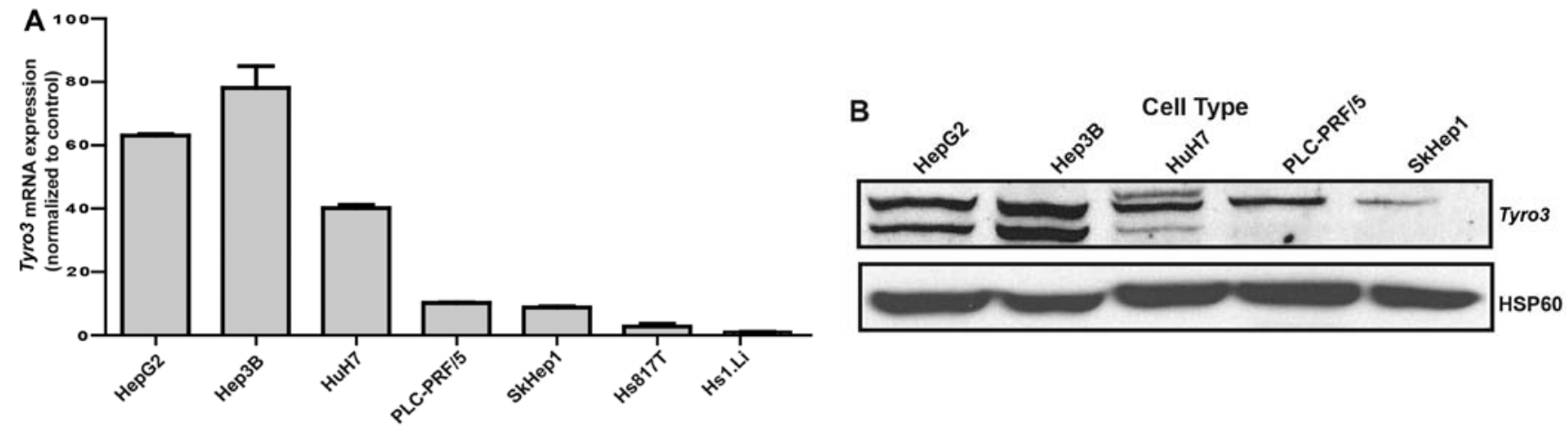

Figure 3. Comparison of tyro3 expression in different liver cancer cell lines. (A) Tyro3 levels in various HCC cell lines are expressed as fold changes relative to $H s$ l.Li as a normal hepatocyte cell line with error bars representing standard deviation (SD) converted to fold changes. (B) Protein expression of tyro3 in selected liver cancer cell lines was determined by immunoblot assay using anti-Tyro3. HSP60 expression was used as a housekeeping control. Hep3B, which exhibited the highest tyro3 expression level, was selected for subsequent studies to establish the role of Tyro3 in HCC progression.

in vitro experiments were pertinent to establishing cause-andeffect relationship and to gain mechanistic understanding into its role. Firstly, a panel of HCC cell lines and normal liver cells were compared in terms of Tyro3 expression (Fig. 3A). By performing quantitative RT-PCR on all the samples, varying transcript levels but generally higher than non-cancerous tissue expression was observed. A HBV-infected HCC cell line, Hep3B was found to be most highly expressing Tyro3 at the transcript and protein level (Fig. 3B). Hence, this cell line was picked for subsequent gene silencing experiments to determine the role of Tyro3 on cancer phenotypes.

Inhibition of Tyro3 expression by siRNA. Hep3B cells were transfected with either scrambled or Tyro3-targeting siRNAs.
Gene silencing was effective in achieving $>80 \%$ reduction in Tyro3 expression (Fig. 4B). Tyro3 knock-down cells demonstrated a suppression of cell viability to $\sim 60 \%$ of scrambled controls, based on intracellular ATP levels (Fig. 4A). To determine if the decimated cell population is due to suppression of cell proliferation, downstream ERK phosphorylation was determined. Based on immunoblot assay, ERK phosphorylation was significantly reduced in Tyro3-silenced cells (Fig. 4B). A corresponding western blot analysis for cyclin D1 also indicated a reduction in the commitment of downstream cell cycling protein. Furthermore, AFP transcript level was determined as a marker of tumor aggressiveness. Here, we report that Tyro3-silenced Hep3B exhibited 50\% reduction in $A F P$ transcript expression as compared to scrambled controls 
Table II. HCC tissue microarray.

\begin{tabular}{|c|c|c|c|c|c|}
\hline & & & $\begin{array}{lllllll}01 & 02 & 03 & 04 & 05 & 06 & 0 \\
10 & 11 & 12 & 13 & 14 & 15 & 1 \\
19 & 20 & 21 & 22 & 23 & 24 & 2 \\
28 & 29 & 30 & 31 & 32 & 33 & 3 \\
37 & 38 & 39 & 40 & 41 & 42 & 4 \\
46 & 47 & 48 & 49 & 50 & 51 & 5 \\
55 & 56 & 57 & 58 & 59 & 60 & 6\end{array}$ & $\begin{array}{lll}07 & 08 & 09 \\
16 & 17 & 18 \\
25 & 26 & 27 \\
34 & 35 & 36 \\
43 & 44 & 45 \\
52 & 53 & 54 \\
61 & 62 & 63\end{array}$ & \\
\hline Layout & Age (years) & Gender & Organ & Pathology diagnosis & Note \\
\hline 1 & 43 & Male & Liver & Hepatocellular carcinoma (grade II) & 0.5 \\
\hline 2 & 39 & Male & Liver & Hepatocellular carcinoma (grade III) & 0 \\
\hline 3 & 55 & Female & Liver & Hepatocellular carcinoma (grade II) & 1 \\
\hline 4 & 32 & Female & Liver & Hepatocellular carcinoma (grade II) & 1 \\
\hline 5 & 57 & Male & Liver & Inflammation & 2.5 \\
\hline 6 & 63 & Male & Liver & Hepatocellular carcinoma (grade II) & 0 \\
\hline 7 & 37 & Female & Liver & Hepatocellular carcinoma (grade II) & 1 \\
\hline 8 & 38 & Male & Liver & Hepatocellular carcinoma (grade II) & 2 \\
\hline 9 & 52 & Female & Liver & Hepatocellular carcinoma (grade II) & 0 \\
\hline 10 & 48 & Male & Liver & Hepatocellular carcinoma (grade II) & 1.5 \\
\hline 11 & 49 & Male & Liver & Hepatocellular carcinoma (grade II) & 0.5 \\
\hline 12 & 43 & Male & Liver & Hepatocellular carcinoma (grade III) & 2 \\
\hline 13 & 36 & Male & Liver & Hepatocellular carcinoma (grade II) & 2 \\
\hline 14 & 45 & Male & Liver & Hepatocellular carcinoma (grade II) & 1 \\
\hline 15 & 71 & Male & Liver & Hepatocellular carcinoma (grade II) & 0 \\
\hline 16 & 43 & Female & Liver & Hepatocellular carcinoma (grade II) & 3 \\
\hline 17 & 52 & Male & Liver & Hepatocellular carcinoma (grade III) & 0 \\
\hline 18 & 49 & Male & Liver & Hepatocellular carcinoma (grade II) & 2 \\
\hline 19 & 63 & Male & Liver & Hepatocellular carcinoma (grade II) & 0 \\
\hline 20 & 63 & Female & Liver & Hepatocellular carcinoma (grade II) & 3 \\
\hline 21 & 46 & Male & Liver & Hepatocellular carcinoma (grade II) & 3 \\
\hline 22 & 48 & Male & Liver & Hepatocellular carcinoma (grade II) & 3 \\
\hline 23 & 49 & Male & Liver & Hepatocellular carcinoma (grade II) & 2 \\
\hline 24 & 48 & Male & Liver & Hepatocellular carcinoma (grade II) & 2 \\
\hline 25 & 43 & Female & Liver & Hepatocellular carcinoma (grade II) & 2 \\
\hline 26 & 67 & Male & Liver & Hepatocellular carcinoma (grade II) & 0.5 \\
\hline 27 & 63 & Male & Liver & Hepatocellular carcinoma (grade II) & 1 \\
\hline 28 & 60 & Male & Liver & Hepatocellular carcinoma (grade II) & 0.5 \\
\hline 29 & 56 & Male & Liver & Hepatocellular carcinoma (grade II) & 2 \\
\hline 30 & 43 & Male & Liver & Hepatocellular carcinoma (grade II) & 2 \\
\hline 31 & 58 & Male & Liver & Hepatocellular carcinoma (grade II) & 3 \\
\hline 32 & 50 & Male & Liver & Hepatocellular carcinoma (grade II) & 2.5 \\
\hline 33 & 35 & Male & Liver & Hepatocellular carcinoma (grade II) & 0 \\
\hline 34 & 61 & Male & Liver & Hepatocellular carcinoma (grade II) & 2 \\
\hline 35 & 50 & Male & Liver & Hepatocellular carcinoma (grade III) & 1 \\
\hline 36 & 62 & Female & Liver & Hepatocellular carcinoma (grade I) & 3 \\
\hline 37 & 39 & Male & Liver & Hepatocellular carcinoma (grade II) & 1 \\
\hline 38 & 67 & Male & Liver & Hepatocellular carcinoma (grade II) & 2 \\
\hline 39 & 37 & Female & Liver & Hepatocellular carcinoma (grade II) & 2 \\
\hline 40 & 62 & Male & Liver & Hepatocellular carcinoma (grade III) & 2 \\
\hline 41 & 50 & Male & Liver & Hepatocellular carcinoma (grade I) & 3 \\
\hline
\end{tabular}


Table II. Continued.

\begin{tabular}{lclllc}
\hline Layout & Age (years) & Gender & Organ & Pathology diagnosis & Note \\
\hline 42 & 41 & Male & Liver & Hepatocellular carcinoma (grade III) & 1.5 \\
43 & 47 & Female & Liver & Hepatocellular carcinoma (grade III) & 2.5 \\
44 & 35 & Male & Liver & Hepatocellular carcinoma (grade II) & 2 \\
45 & 47 & Male & Liver & Hepatocellular carcinoma (grade II) & 2 \\
46 & 56 & Male & Liver & Hepatocellular carcinoma (grade II) & 1 \\
47 & 46 & Male & Liver & Hepatocellular carcinoma (grade I) & 2 \\
48 & 62 & Female & Liver & Inflammation & 1 \\
49 & 77 & Male & Liver & Hepatocellular carcinoma (grade II) & 2.5 \\
50 & 64 & Male & Liver & Hepatocellular carcinoma (grade III) & 2 \\
51 & 35 & Male & Liver & Hepatocellular carcinoma (grade III) & 1 \\
52 & 98 & Female & Liver & Hepatocellular carcinoma (grade II) & 3 \\
53 & 69 & Female & Liver & Hepatocellular carcinoma (grade II) & 2 \\
54 & 58 & Male & Liver & Hepatocellular carcinoma (grade I) & 2 \\
55 & 62 & Male & Liver & Hepatocellular carcinoma (grade II) & 2 \\
56 & 55 & Male & Liver & Inflammation & 2 \\
57 & 53 & Male & Liver & Hepatocellular carcinoma (grade II) & 2 \\
58 & 47 & Male & Liver & Hepatocellular carcinoma (grade III) & 2 \\
59 & 55 & Male & Liver & Hepatocellular carcinoma (grade III) & 1 \\
60 & 57 & Male & Liver & Hepatocellular carcinoma (grade II) & 2 \\
61 & 42 & Female & Liver & Normal tissue & 0.5 \\
62 & 66 & Female & Liver & Normal tissue & 3 \\
63 & 55 & Male & Liver & Normal tissue & 2 \\
\hline
\end{tabular}

(Fig. 4C). Protein expression was also significantly suppressed, in agreement with the observation made at the transcript level (Fig. 4D).

\section{Discussion}

The principle of molecular targeted therapy for cancer treatment is aimed at arresting key signaling mechanism that drives the disease phenotype. In the case of $\mathrm{HCC}$, such a target remains elusive. To this end, we have focused on a relatively uncharacterized tyrosine kinase, Tyro3, of the TAM family. We envisioned that any link between increased Tyro3 expression and disease manifestation will provide important basis and direction for subsequent elucidation of mechanism as well as further characterization of Tyro3 as a drug target.

The present study yielded a number of key findings that qualified the potential role of Tyro3 in tumor progression. The survey of $55 \mathrm{HCC}$ patients revealed that almost half of them exhibit a significant elevation of Tyro3 transcript expression in tumor as compared to the adjacent normal tissue. This finding suggests the possibility that increased Tyro3 may be implicated in the process of normal-to-tumor transition. This is the first time that Tyro3 is reported to be overexpressed in HCC, even though upregulation of Tyro3 has been seen in other malignancies. In one study, majority of the lung carcinoma cell lines investigated revealed co-expression of Tyro3 and its ligand, Protein S, suggesting that an activated Tyro3-signaling mechanism was at work in this cancer type (13). The oncogenic potential of activated Tyro3 was also described in a founding work whereby enforced expression in murine NIH3T3 cells resulted in ligand-independent activation and transformation of the cells into a malignant phenotype (16). Conceivably, an increased expression of Tyro3 within the tumor tissue of HCC would constitute key signaling aberrations that drive tumor progression.

Histologically, we observed that Tyro3 is most strongly stained in the blood cells within the tumor samples of HCC grade II. Comparatively, the intensity of staining was not seen in other types of liver conditions such as vacuolation and necrosis. This supports that Tyro3 overexpression may have a more dominant presence in HCC compared to normal liver as well as other liver pathologies, but the source of its biosynthesis may include both parenchymal and non-parenchymal cells within the liver.

The frequent elevation of Tyro3 observed in the present study justified further characterization because any clinical impact derived may have implications on many HCC patients. Hence, to obtain clinical evidence that Tyro3 expression may affect disease manifestation, we performed statistical analysis using the available clinico-pathological data (15). We found that increased Tyro3 overexpression associated with higher levels of hepatocellular injury, as indicated by ALT elevations. A spike in this serum marker is suggestive of a worse condition of HCC, where liver dysfunction exerts an additional insult that compromises the well-being of the patients. Another liver injury marker AST showed borderline significance but 

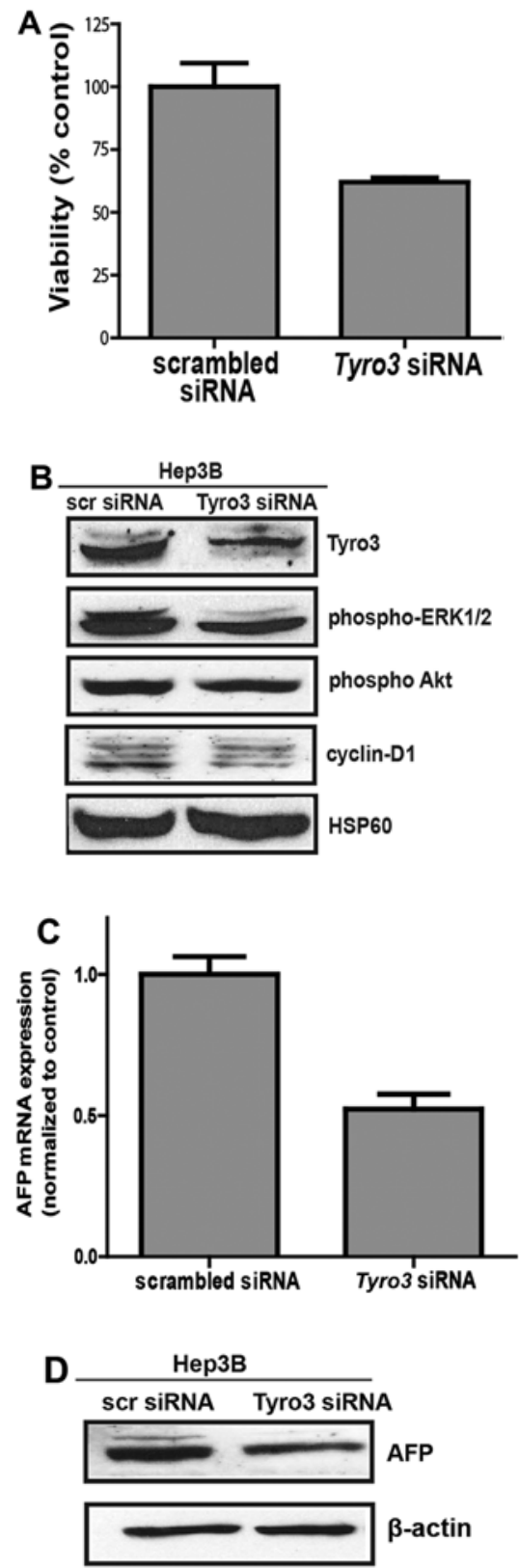

Figure 4. Effect of Tyro3 silencing on cell viability, proliferation and AFP expression. Hep3B cells $\left(1 \times 10^{5} /\right.$ well $)$ were transfected with $100 \mathrm{nM}$ scrambled control or tyro3-targeting siRNA. (A) At $48 \mathrm{~h}$ post transfection, Hep3B cells were re-seeded with 8 replicates $\left(1 \times 10^{4} /\right.$ well $)$ in a 96 -well plate. After incubating for additional $24 \mathrm{~h}$, cell viability was measured using CellTiter-Glo. Results are expressed as a percentage of cell viability in scrambled-treated controls. Error bars indicate coefficient of variation $(n=8)$. (B) At $72 \mathrm{~h}$ post-transfection with siRNA, samples were immunoblotted with anti-Tyro3, anti-pERK, anti-AKT and anti-cyclin D1. HSP60 expression was used as a loading control. (C) gene expression of $a f p$ was determined by qRTCPR and normalized to gapdh. Data indicate fold change in gene expression with respect to scrambled siRNA. Error bars represent SD converted to fold change. (D) Corresponding AFP protein expression was also determined by immunoblot assay using anti- $\beta$-actin as a loading control.

this is deemed as a less selective marker as it responds to muscular injury as well. Separately, higher Tyro3 levels were also associated with higher AFP levels. The exact role of AFP in HCC is not known, even though it is known that AFP is elevated in $\sim 60-70 \%$ of all HCC patients (17). In such patients, higher level of AFP is frequently associated with a greater severity of the disease (18). Another key observation from our data was the association with larger tumor size at the point of surgical resection among the Tyro3 overexpressed patients. A larger tumor underpins greater growth potential of the cancerous cells. That said, subsequent multivariate analyses only substantiated the association between AFP and Tyro3, while other associations with ALT, AST and tumor size were partially accounted for by elevated levels of Tyro3. Overall, the combination of these clinical correlations indicates that Tyro3 overexpression predicts a more aggressive cancer phenotype that warrants further work to establish causality.

We performed in vitro studies to investigate the direct consequence of suppressing Tyro3-mediated signaling in HCC. This would enable us to determine the functional role of Tyro3 in HCC as well as to gain mechanistic insights for the phenotype it generates. Accordingly, Hep3B, an HBV-infected $\mathrm{HCC}$ cell line was found to be suitable for this purpose with several-fold higher Tyro3 levels than non-cancerous liver cell line. We also note that most HCC cell lines exhibited high levels of expression, in agreement with the cancer tissue expression as documented by Human Protein Atlas (http:// www.proteinatlas.org).

Tyro3 knockdown studies in Hep3B cells were aimed to address two major phenomena derived from the clinical data: Tyro3 overexpression association with enhanced cell growth (larger tumor diameter) and tumor aggressiveness (AFP expression). Clearly, Tyro3 suppression in HCC cell line effectively reduced cell viability. The inhibition of downstream ERK phosphorylation and cyclin D1 suggest that Tyro3 may at least in part, mediate MAPK signaling to elicit cell proliferation to support tumor growth and proliferation. This observation is consistent with a report from another group who found that binding of the ligand, Gas6, to Tyro3 activated MAPK signaling and activity in osteoclastic cells (19). Separately, to clarify the effect of Tyro3 on AFP, we performed quantification of AFP transcript and protein after Tyro3-silencing. AFP suppression was significant, suggesting that Tyro3 likely exerted additional biochemical effects independent of cell proliferation in HCC cell lines. This latter effect deserves further investigation as it implies inhibition of Tyro3 may confer therapeutic advantage through an alteration of the aggressive potential of the HCC, besides just arresting tumor growth.

While we have shown that the effect of Tyro3 signaling was transmitted through ERK phosphorylation, other pathways cannot be ignored. Previous studies have reported the co-localization of Tyro3 with non-receptor tyrosine kinases of the SRC family (20). PI3K has also been described to be identified as another interacting partner with Tyro3 from yeast-two-hybrid screen (21). More recently, Tyro3 was shown to induce melanocyte-specific microphthalmia-associated transcription factor (MITF-M) expression and may play a role in melanoma development through a signaling pathway that is involved in this pathway (22). These pathways provide specific directions to establish the biochemical mechanism linking aberrations in Tyro3 expression to the onset of cancer phenotypes that we have observed. Such studies will ascertain the therapeutic value of targeting Tyro3 for the effective management of HCC.

In conclusion, this study uncovers the frequent overexpression of Tyro3 in HCC and lay important groundwork 
for further investigation of its role in the propagation of an aggressive cancer phenotype. Clinically, the present study could be expanded to differentiate the involvement of Tyro3 in histologically and etiologically distinct HCC. The experimental models used in this study can also be exploited further to gain more insights into the mechanism of action of Tyro3. Without negating the possibility of receptor crosstalk and the involvement of other kinases in cancer, one should note that the role of Tyro3 should be cross-examined in the context of other tyrosine kinases also involved in $\mathrm{HCC}$ so that its relative importance and the feasibility of combinational treatment can be considered suitably.

\section{Acknowledgements}

The present study was supported by the NUS Grant R-148000-187-112 (to H.K.H), the Biomedical Research Council intramural grant (to B.T.C.), and by the NUS Graduate Scholarship (to Y.D).

\section{References}

1. El-Serag HB and Rudolph KL: Hepatocellular carcinoma: Epidemiology and molecular carcinogenesis. Gastroenterology 132: 2557-2576, 2007.

2. Llovet JM, Ricci S, Mazzaferro V, Hilgard P, Gane E, Blanc JF, de Oliveira AC, Santoro A, Raoul JL, Forner A, et al; SHARP Investigators Study Group: Sorafenib in advanced hepatocellular carcinoma. N Engl J Med 359: 378-390, 2008.

3. Huynh H: Tyrosine kinase inhibitors to treat liver cancer. Expert Opin Emerg Drugs 15: 13-26, 2010.

4. Olsen SK, Brown RS and Siegel AB: Hepatocellular carcinoma: Review of current treatment with a focus on targeted molecular therapies. Therap Adv Gastroenterol 3: 55-66, 2010.

5. Whittaker S, Marais R and Zhu AX: The role of signaling pathways in the development and treatment of hepatocellular carcinoma. Oncogene 29: 4989-5005, 2010.

6. Hsu C, Yang TS, Huo TI, Hsieh RK, Yu CW, Hwang WS, Hsieh TY, Huang WT, Chao Y, Meng R, et al: Vandetanib in patients with inoperable hepatocellular carcinoma: A phase II, randomized, double-blind, placebo-controlled study. J Hepatol 56: 1097-1103, 2012

7. Lin AY, Fisher GA, So S, Tang C and Levitt L: Phase II study of imatinib in unresectable hepatocellular carcinoma. Am J Clin Oncol 31: 84-88, 2008.

8. Faivre S, Raymond E, Boucher E, Douillard J, Lim HY, Kim JS, Zappa M, Lanzalone S, Lin X, Deprimo S, et al: Safety and efficacy of sunitinib in patients with advanced hepatocellular carcinoma: An open-label, multicentre, phase II study. Lancet Oncol 10: 794-800, 2009

9. Linger RM, Keating AK, Earp HS and Graham DK: TAM receptor tyrosine kinases: Biologic functions, signaling, and potential therapeutic targeting in human cancer. Adv Cancer Res 100: $35-83,2008$.
10. Zhang YX, Knyazev PG, Cheburkin YV, Sharma K, Knyazev YP, Orfi L, Szabadkai I, Daub H, Kéri G and Ullrich A: AXL is a potential target for therapeutic intervention in breast cancer progression. Cancer Res 68: 1905-1915, 2008

11. Taylor IC, Roy S, Yaswen P, Stampfer MR and Varmus HE: Mouse mammary tumors express elevated levels of RNA encoding the murine homology of SKY, a putative receptor tyrosine kinase. J Biol Chem 270: 6872-6880, 1995.

12. De Vos J, Couderc G, Tarte K, Jourdan M, Requirand G Delteil MC, Rossi JF, Mechti N and Klein B: Identifying intercellular signaling genes expressed in malignant plasma cells by using complementary DNA arrays. Blood 98: 771-780, 2001.

13. Wimmel A, Rohner I, Ramaswamy A, Heidtmann HH, Seitz R, Kraus $M$ and Schuermann M: Synthesis and secretion of the anticoagulant protein $\mathrm{S}$ and coexpression of the Tyro3 receptor in human lung carcinoma cells. Cancer 86: 43-49, 1999.

14. Ho HK, Chua BT, Wong W, Lim KS, Teo V, Ong HT, Chen X, Zhang W, Hui KM, Go ML, et al: Benzylidene-indolinones are effective as multi-targeted kinase inhibitor therapeutics against hepatocellular carcinoma. Mol Oncol 8: 1266-1277, 2014.

15. Ho HK, Pok S, Streit S, Ruhe JE, Hart S, Lim KS, Loo HL, Aung MO, Lim SG and Ullrich A: Fibroblast growth factor receptor 4 regulates proliferation, anti-apoptosis and alpha-fetoprotein secretion during hepatocellular carcinoma progression and represents a potential target for therapeutic intervention. J Hepatol 50: 118-127, 2009.

16. Taylor IC, Roy S and Varmus HE: Overexpression of the Sky receptor tyrosine kinase at the cell surface or in the cytoplasm results in ligand-independent activation. Oncogene 11:2619-2626, 1995.

17. Abelev GI and Eraiser TL: Cellular aspects of alpha-fetoprotein reexpression in tumors. Semin Cancer Biol 9: 95-107, 1999.

18. Vibert E, Azoulay D, Hoti E, Iacopinelli S, Samuel D, Salloum C, Lemoine A, Bismuth H, Castaing D and Adam R: Progression of alphafetoprotein before liver transplantation for hepatocellular carcinoma in cirrhotic patients: a critical factor. Am J Transplant 10: 129-137, 2010.

19. Katagiri M, Hakeda Y, Chikazu D, Ogasawara T, Takato T, Kumegawa M, Nakamura K and Kawaguchi H: Mechanism of stimulation of osteoclastic bone resorption through Gas6/Tyro 3, a receptor tyrosine kinase signaling, in mouse osteoclasts. J Biol Chem 276: 7376-7382, 2001.

20. Toshima J, Ohashi K, Iwashita S and Mizuno K: Autophosphorylation activity and association with Src family kinase of Sky receptor tyrosine kinase. Biochem Biophys Res Commun 209: 656-663, 1995.

21. Lan Z, Wu H, Li W, Wu S, Lu L, Xu M and Dai W: Transforming activity of receptor tyrosine kinase tyro3 is mediated, at least in part, by the PI3 kinase-signaling pathway. Blood 95: 633-638, 2000.

22. Zhu S, Wurdak H, Wang Y, Galkin A, Tao H, Li J, Lyssiotis CA, Yan F, Tu BP, Miraglia L, et al: A genomic screen identifies TYRO3 as a MITF regulator in melanoma. Proc Natl Acad Sci USA 106: 17025-17030, 2009. 